\title{
Some Mycetozoa (Myxomycetes) Members from Zorkun High Plateau (Osmaniye)
}

\author{
Hayri BABA \\ Mustafa Kemal University, Faculty of Science \& Arts, Biology Department, 31040, Antakya-Hatay, Turkey \\ hayribaba_68@hotmail.com
}

\section{Zorkun Yaylası (Osmaniye)'ndan Bazı Mycetozoa (Myxomycetes) Üyeleri}

\begin{abstract}
Myxomycete samples collected from 4 different localities from Zorkun Plateau (Osmaniye-Turkey) between 20142016. As a result of field and laboratory studies 28 species belonging to 9 families and 16 genera were determined. Among them, seven species were collected only from nature, 16 were obtained by Moist Chamber Technique in laboratory, and 5 species were obtained both from nature and by Moist Chamber Technique. Echinostelium minutum, Cribraria cancellata, Didymium difforme, Didymium squamulosum, Physarum album, and Arcyria cinerea are the most common species. Ten species were found to be common and 12 are rare or occasional. Species/genus ratio $(\mathrm{S} / \mathrm{G})$ is 1.75 . The determined species are given together with habitats, substrates, coordinates, collection dates, voucher numbers and prevalencies.
\end{abstract}

Key words: Myxomycete, Zorkun Hihgh Plateau, Osmaniye, Turkey

Özet: Miksomiset örnekleri 2014-2016 yılları arasında Zorkun Yaylasından (Osmaniye-Türkiye) 4 farklı lokaliteden toplandı. Arazi ve laboratuvar çalışmaları sonucunda 9 familya ve 16 cinse ait 28 tür tespit edilmiştir. Toplanan örneklerden 7 tür sadece doğal ortamdan, 16 tür Nem Odası Tekniği ilelaboratuvar şartlarında ve 5 tür ise hem doğal ortamdan, hem de Nem Odası Tekniği ile elde edilmiştir. Echinostelium minutum, Cribraria cancellata, Didymium difforme, Didymium squamulosum, Physarum album, ve Arcyria cinerea en yaygın türlerdir. On tür yaygın, 12 tür de arasıra görülen olarak belirlenmiștir. Tür/Cins oranı 1.75 olarak bulunmuştur. Belirlenen türler habitat, substrat, konum koordinatı, toplanma tarihi, toplayıcı numaraları ve yaygınlık durumları ile birlikte listelenmiş̧ir.

Anahtar Kelimeler: Miksomiset, Zorkun Yaylası, Osmaniye, Türkiye

\section{Introduction}

Mycetozoa; plasmodial slime moulds; Myxomycetes; a small group of organisms with amorphous, multinucleate and protoplasmic mass and about 994 species world-wide (Lado, 2017). Two hundred and sixty two 262 species have been reported from Turkey (Sesli et al., 2016). Molecular and physiological studies support the classification of myxomycetes within eukaryotic organisms in the kingdom Protoctista (Everhart and Keller, 2008). Plasmodial slime molds are generally found on decaying or living plant materials. Mostly cosmopolitan, several microhabitats for these species have also been reported in forest ecosystems.

Osmaniye is situated in the Eastern Mediterranean region of Turkey with a surface area of $3280 \mathrm{~km}^{2}$, and surrounded by Gaziantep to the east, Hatay to the south, Adana to the west and Kahramanmarass to the north. Southeastern parts are also bordered by the Amanos Mountains, extending from the port of Iskenderun to the east, while northwestern parts are bordered by Toros Mountains. Osmaniye has a Mediterranean climate. Summers are very hot and dry while the winters are cool and wet (Anonymous, 2017). The Zorkun high plateau is located in the most eastern side of the Mediterranean region within Osmaniye province (Turkey) (Figure 1). It is one of the most important and old high plateau of Osmaniye and Çukurova region at the northern slopes of the Amanos mountains. Its population reaches almost to 60.000 people during summer. Average altitude is about 1.550 meters. The Zorkun high plateau is of significant research interest because of different ecosystem types in both forests and agricultural lands. The highest point of the plaeau is Keldaz hill $(2108 \mathrm{~m})$. The vegetation of
Zorkun plateau is very rich; Pinus brutia and maquis are mixed with up to 600 meters. Pinus brutia forests are dominant between 600-900 meters. Between 900-1100 meters mixed populations of Pinus nigra and Pinus brutia are visible, together with Carpinus orientalis, Buxus sempervirens along stream sides. After 1300 meters Quercus infectoria takes the place of $P$. brutia together with Pinus nigra. Between the altitudes of 1400- 1800 meters, Pinus nigra is the most dominated tree in the region. Fagus orientalis, is distributed among $P$. nigra above 1800 meters. Some pure Abies cilicica populations also exist between 1900-2000 meters (Sevgi, 1984; Gürçınar and Yüceer, 1995).

The work aims to make a contribution to the myxobiota of Turkey.

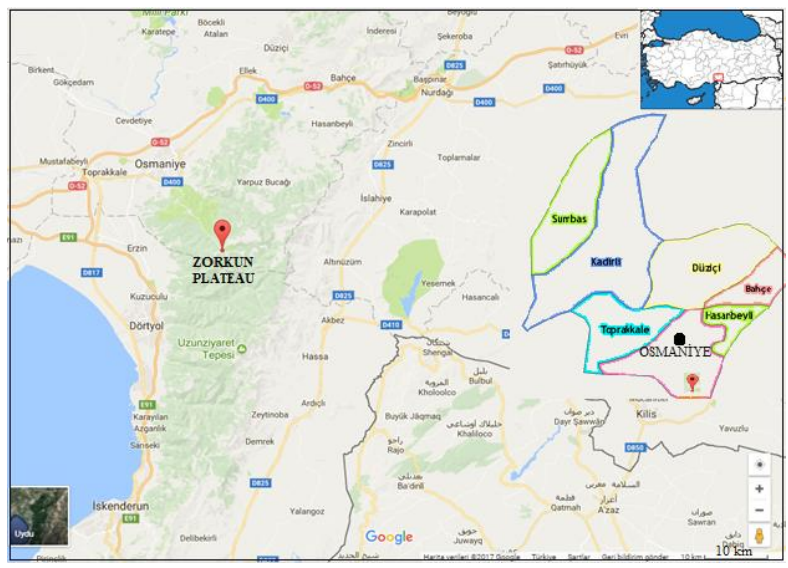

Figure 1. Map of Osmaniye and Zorkun high plateau 


\section{Materials and Method}

Natural substrates and debris material, like bark of living trees, as well as decaying bark, wood, leaves and litter were collected from four different stations in Zorkun Hihgh Plateau. Natural mature fructifications were directly collected from the substratum and placed in cardboard herbarium boxes. Fructifications of some myxomycetes were obtained from the moist chamber culture in the laboratory. Collected plants materials were moistened with distilled water. The moist chambers were examined every day under a stereo-microscope. When fructification of myxomycetes were detected in the culture, the moist chamber was allowed to dry slowly and the myxomycetes were then dried for one week and prepared as fungarium material.

Microscopic and macroscopic features of the samples were determined in the laboratory. The morphological characters; fruiting bodies shape, size and colour, spore size and ornamentation, capillitium colour and branching, lime crystalsize and morphology, stalk colour and proportion were measured. The specimens were identified according to the relevant references (Martin and Alexopoulos, 1969; Neubert et al., 1993, 1995 and 2000). The samples are stored in fungarium of biology department.

\section{Results}

In this study 28 species (65 samples) belonging to 6 orders, 9 families and 16 genera were identified. Twenty three samples ( 7 species) were collected in field, 42 samples (16 species) were developed in moist chamber culture and 5 species were both collected from nature and obtained by moist chamber culture in laboratory.

Abundance indices were applied to all species collected from research area. These indices are categorised as Rare (for species $<0.5 \%$ of total number collections), Occasional (for species $>0.5 \%$ but $<1.5 \%$ of total), Common (for species $>1.5 \%$ but $<3.0 \%$ of total), and Abundant (for species $>3.0 \%$ of total) (Stephenson et al., 1993). In our study, 6 species were found to be abundant (A), 10 species were common (C), 12 species were occasional $(\mathrm{O})$. The mean number of species per genus $(\mathrm{S} / \mathrm{G})$ was calculated from the data sets of study area as 1.75 .

Mycetozoa

Protostelia

Protosteliida

Ceratiomyxaceae

1. Ceratiomyxa fruticulosa (O.F. Müll.) T. Macbr.

Derviş Pınarı, on conifer wood, $1500 \mathrm{~m}, 36^{\circ} 58^{\prime} 15^{\prime \prime} \mathrm{N}$; $36^{\circ} 0^{\prime} 56^{\prime \prime}$ E, 06.11.2014, Baba 18; Çift Mazı, on woody debris, $800 \mathrm{~m}, 37^{\circ} 01^{\prime} 10^{\prime \prime} \mathrm{N}$; 36 $16^{\prime} 17^{\prime \prime} \mathrm{E}, 26.01 .2015$, Natural, Baba 21, Common.

\section{Myxogastria \\ Echinosteliida \\ Echinosteliaceae}

\section{Echinostelium minutum de Bary}

Derviş Pınarı, on wood, $1500 \mathrm{~m}, 36^{\circ} 58^{\prime} 15^{\prime \prime} \mathrm{N} ; 36^{\circ} 0^{\prime} 56^{\prime \prime} \mathrm{E}$, 06.11.2014, Baba 2, 17, 45; Kent Orman1, on Pinus sp. cone, $800 \mathrm{~m}, 37^{\circ} 01^{\prime} 19^{\prime \prime} \mathrm{N}$; 36 $16^{\prime} 30^{\prime \prime} \mathrm{E}, 26.01 .2015$, Baba
11; Cift Mazı, on Pinus sp. bark, $800 \mathrm{~m}, 37^{\circ} 01^{\prime} 10^{\prime \prime} \mathrm{N}$ $36^{\circ} 18^{\prime} 17^{\prime \prime} \mathrm{E}, 26.01 .2015$, Baba 2, Abundant.

\section{Liceida \\ Cribrariaceae}

3. Cribraria cancellata (Batsch) Nann.-Bremek.

Keldaz hill, on woods, $2100 \mathrm{~m}, 36^{\circ} 51^{\prime} 12^{\prime \prime} \mathrm{N}-36^{\circ} 22^{\prime} 58^{\prime \prime} \mathrm{E}$, 06.11.2014, Natural, Baba 19, 40, 41, Abundant.

\section{Cribraria microcarpa (Schrad.) Pers.}

Kent Orman1, on wood, $800 \mathrm{~m}, 37^{\circ} 01^{\prime} 19^{\prime \prime} \mathrm{N}-36^{\circ} 16^{\prime} 30^{\prime \prime} \mathrm{E}$, 26.01.2015, Baba 24; Derviş Pınarı, on conifer bark, 1500 $\mathrm{m}, 36^{\circ} 58^{\prime} 15^{\prime \prime} \mathrm{N}-36^{\circ} 0^{\prime} 56^{\prime \prime} \mathrm{E}, 06.11 .2014$, Natural, Baba 30, Common.

\section{Cribraria violacea $\mathrm{Rex}$}

Çift Mazı, on conifer wood, $800 \mathrm{~m}, 37^{\circ} 01^{\prime} 10^{\prime \prime} \mathrm{N}-$ $36^{\circ} 18^{\prime} 17^{\prime \prime}$ E, 26.01.2015, Baba 11, Occasional.

\section{Liceaceae}

\section{Licea biforis Morgan}

Kent Orman1, on Pinus sp. bark, 800 m, 37 $01^{\prime} 19^{\prime \prime} \mathrm{N}-$ $36^{\circ} 16^{\prime} 30^{\prime \prime} \mathrm{E}, 06.11 .2014$, Baba 26. Occasional.

\section{Licea castanea G.Lister}

Derviş Pınarı, on conifer bark, $1500 \mathrm{~m}, 36^{\circ} 58^{\prime} 15^{\prime \prime} \mathrm{N}$ $36^{\circ} 0^{\prime} 56^{\prime \prime} \mathrm{E}, 06.11 .2014$, Baba 30, Occasional.

\section{Licea minima Fr.}

Keldaz hill, on conifer barks, $2100 \mathrm{~m}, 36^{\circ} 51^{\prime} 12^{\prime \prime} \mathrm{N}$ $36^{\circ} 22^{\prime} 58^{\prime \prime}$ E, 06.11.2014, Baba 9, 48, Common.

\section{Reticulariaceae}

9. Lycogala epidendrum (J.C.Buxb. ex L.) Fr.

Çift Mazı, on decaying bark, $800 \mathrm{~m}, 37^{\circ} 01^{\prime} 10^{\prime \prime} \mathrm{N}$ $36^{\circ} 18^{\prime} 17^{\prime \prime}$ E, 26.01.2015, Natural, Baba 10, Occasional.

\section{Physarida \\ Didymiaceae}

\section{Didymium difforme (Pers.) S.F.Gray}

Kent Orman1, in coniferous debris leaves, $800 \mathrm{~m}$, $37^{\circ} 01^{\prime} 19^{\prime \prime} \mathrm{N}-36^{\circ} 16^{\prime} 30^{\prime \prime} \mathrm{E}, 26.01 .2015$, Baba 21, 29, 36, 47; Kent orman1, on pomegranate bark, $800 \mathrm{~m}, 37^{\circ} 01^{\prime} 19^{\prime \prime} \mathrm{N}$ $36^{\circ} 16^{\prime} 30^{\prime \prime}$ E, 26.01.2015, Natural, Baba 28; Çift Mazı, on leaves, $800 \mathrm{~m}, 37^{\circ} 01^{\prime} 10^{\prime \prime} \mathrm{N}-36^{\circ} 18^{\prime} 17^{\prime \prime} \mathrm{E}, 26.01 .2015$, Baba 54, 78; Derviş Pınarı, on conifer leaves, 1500 meter, $36^{\circ} 58^{\prime} 15^{\prime \prime} \mathrm{N}-36^{\circ} 0^{\prime} 56^{\prime \prime} \mathrm{E}, 26.01 .2015$, Natural, Baba 22, 88, Abundant.

\section{Didymium squamulosum (Alb. \& Schwein.) Fr.}

Çift Mazı, on leaves, $800 \mathrm{~m}, 37^{\circ} 01^{\prime} 10^{\prime \prime} \mathrm{N}-36^{\circ} 18^{\prime} 17^{\prime \prime} \mathrm{E}$, 26.01.2015, Baba 54, 78; Derviş Pınarı, on conifer leaves, $1500 \mathrm{~m}, 36^{\circ} 58^{\prime} 15^{\prime \prime} \mathrm{N}-36^{\circ} 0^{\prime} 56^{\prime \prime} \mathrm{E}, 26.01 .2015$, Baba 22, 88, Abundant.

\section{Physaraceae}

\section{Physarum album (Bull.) Chevall.}

Çift Mazı, on woods, $800 \mathrm{~m}, 37^{\circ} 01^{\prime} 10^{\prime \prime} \mathrm{N}-36^{\circ} 18^{\prime} 17^{\prime \prime} \mathrm{E}$, 26.01.2015, Natural, Baba 4, 71; Derviş Pınarı, on conifer barks, $1500 \mathrm{~m}, 36^{\circ} 58^{\prime} 15^{\prime \prime} \mathrm{N}-36^{\circ} 0^{\prime} 56^{\prime \prime} \mathrm{E}, 26.01 .2015$, Baba 12, 18, Abundant. 
13. Physarum cinereum (Batsch) Pers.

Çift Mazı, on debris barks, $800 \mathrm{~m}, 37^{\circ} 01^{\prime} 10^{\prime \prime} \mathrm{N}-$ $36^{\circ} 18^{\prime} 17^{\prime \prime}$ E, 26.01.2015, Baba 54, 78. Common.

14. Fuligo septica (L.) F.H. Wigg.

Kent orman1, on grass leaves, $800 \mathrm{~m}, 37^{\circ} 01^{\prime} 19^{\prime \prime} \mathrm{N}-$ $36^{\circ} 16^{\prime} 30^{\prime \prime}$ E, 26.01.2015, Natural, Baba 51, 88, Common.

\section{Stemonitida}

\section{Stemonitidaceae}

15. Collaria lurida (Lister) Nann.-Bremek.

Çift Mazı, on conifer barks, $800 \mathrm{~m}, 37^{\circ} 01^{\prime} 10^{\prime \prime} \mathrm{N}-$ $36^{\circ} 18^{\prime} 17^{\prime \prime}$ E, 26.01.2015, Baba 8, 28, Common.

16. Comatricha ellae Härk.

Derviş Pınarı, on conifer barks, $1500 \mathrm{~m}, 36^{\circ} 58^{\prime} 15^{\prime \prime} \mathrm{N}$ $36^{\circ} 0^{\prime} 56^{\prime \prime} \mathrm{E}, 26.01 .2015$, Baba 41, Occasional.

\section{Comatricha pulchella (C. Bab.) Rostaf.}

Derviş Pınarı, on coniferous leaves, $1500 \mathrm{~m}, 36^{\circ} 58^{\prime} 15^{\prime \prime} \mathrm{N}-$ $36^{\circ} 0^{\prime} 56^{\prime \prime} \mathrm{E}, 26.01 .2015$, Natural, Baba 26, Occasional.

\section{Comatricha nigra (Pers. ex J.F. Gmel.) J. Schröt.}

Derviş Pınarı, on conifer woods, $1500 \mathrm{~m}, 36^{\circ} 58^{\prime} 15^{\prime \prime} \mathrm{N}-36^{\circ} 0^{\prime}$ 6"E, 26.01.2015, Baba 2, Occasional.

19. Enerthenema papillatum (Pers.) Rostaf.

Derviş Pınarı, on conifer woods, $1500 \mathrm{~m}, 36^{\circ} 58^{\prime} 15^{\prime \prime} \mathrm{N}-$ $36^{\circ} 0^{\prime} 56^{\prime \prime} \mathrm{E}, 26.01 .2015$, Natural, Baba 8, Occasional.

20. Macbrideola cornea (G. Lister \& Cran) Alexop.

Derviş Pınarı, on conifer barks, $1500 \mathrm{~m}, 36^{\circ} 58^{\prime} 15^{\prime \prime} \mathrm{N}-$ $36^{\circ} 0^{\prime} 56^{\prime \prime} \mathrm{E}, 26.01 .2015$, Baba 10, Occasional.

21. Stemonitopsis amoena (Nann.-Bremek.) Nann.Bremek.

Derviş Pınarı, on conifer woods, $1500 \mathrm{~m}, 36^{\circ} 58^{\prime} 15^{\prime \prime} \mathrm{N}-$ $36^{\circ} 0^{\prime} 56^{\prime \prime} \mathrm{E}, 26.01 .2015$, Baba 22; Çift Mazı, on barks, 800 $\mathrm{m}, \quad 37^{\circ} 01^{\prime} 10^{\prime \prime} \mathrm{N}-36^{\circ} 18^{\prime} 17^{\prime \prime} \mathrm{E}, 26.01 .2015$, Baba 12, Common.

\section{Trichiida}

\section{Arcyriaceae}

22. Arcyria cinerea (Bull.) Pers.

Derviş Pınarı, on conifer woods, $1500 \mathrm{~m}, 36^{\circ} 58^{\prime} 15^{\prime \prime} \mathrm{N}-$ $36^{\circ} 0^{\prime} 56^{\prime \prime} \mathrm{E}$, 26.01.2015, Natural, Baba 22, 47, 58; Çift Maz1, on barks, $800 \mathrm{~m}, 37^{\circ} 01^{\prime} 10^{\prime \prime} \mathrm{N}-36^{\circ} 18^{\prime} 17^{\prime \prime} \mathrm{E}$, 26.01.2015, Natural, Baba 12, 56; Keldaz hill, on conifer barks, $2100 \mathrm{~m}, 36^{\circ} 51^{\prime} 12^{\prime \prime} \mathrm{N}-36^{\circ} 22^{\prime} 58^{\prime \prime} \mathrm{E}, 06.11 .2014$, Baba 9, 48, 59, Abundant.

\section{Arcyria incarnata (Pers.) Pers.}

Derviş Pınarı, on conifer woods, $1500 \mathrm{~m}, 36^{\circ} 58^{\prime} 15^{\prime \prime} \mathrm{N}-$ $36^{\circ} 0^{\prime} 56^{\prime \prime} \mathrm{E}, 26.01 .2015$, Baba 22, Occasional.

24. Arcyria pomiformis (Leers) Rostaf.

Derviş Pınarı, on conifer woods, $1500 \mathrm{~m}, 36^{\circ} 58^{\prime} 15^{\prime \prime} \mathrm{N}-$ $36^{\circ} 0^{\prime} 56^{\prime \prime} \mathrm{E}$, 26.01.2015, Baba 22; Çift Mazı, on debris barks, $800 \mathrm{~m}, \quad 37^{\circ} 01^{\prime} 10^{\prime \prime} \mathrm{N}-36^{\circ} 18^{\prime} 17^{\prime \prime} \mathrm{E}$, Baba 26, Common.

\section{Arcyria obvelata (Oeder) Onsberg}

Çift Mazı, on woody debris, $800 \mathrm{~m}, 37^{\circ} 01^{\prime} 10^{\prime \prime} \mathrm{N}$; $36^{\circ} 18^{\prime} 17^{\prime \prime}$ E, 26.01.2015, Natural, Baba 54, 78, Common.

\section{Trichiaceae}

26. Perichaena corticalis (Batsch ) Rostaf.

Kent orman1, on debris barks, $800 \mathrm{~m}, 37^{\circ} 01^{\prime} 19^{\prime \prime} \mathrm{N}-$ $36^{\circ} 16^{\prime} 30^{\prime \prime} \mathrm{E}, 26.01 .2015$, Baba 54, 78, Common.

\section{Perichaena depressa Lib.}

Çift Mazı, on debris barks, $800 \mathrm{~m}, 37^{\circ} 01^{\prime} 10^{\prime \prime} \mathrm{N}$ $36^{\circ} 18^{\prime} 17^{\prime \prime}$ E, 26.01.2015, Baba 42, Occasional.

\section{Trichia decipiens (Pers.) T. Macbr.}

Çift Mazı, on debris, $800 \mathrm{~m}, 37^{\circ} 01^{\prime} 10^{\prime \prime} \mathrm{N}-36^{\circ} 18^{\prime} 17^{\prime \prime} \mathrm{E}$, 26.01.2015, Natural, Baba 5, Occasional.

\section{Discussions}

Our research is the first study on myxomycetes of Osmaniye. Totaly 28 species were determined for Osmaniye myxobiota. Echinostelium minutum, Cribraria cancellata, Didymium difforme, Didymium squamulosum, Physarum album, and Arcyria cinerea are the most common species (Abundant) in research area, and they comprised the $50.7 \%$ of all the samples. Many studies have also reported that the above-mentioned species are cosmopolitan on different substrates (Alexopoulos et al., 1996; Ergül and Akgül, 2011; Stephenson, 2014; Baba et al., 2016). The most common genera to be determined in the region was Arcyria. Then comes Comatricha, Didymium, Echinostelium and Physarum. Comprising 5 genera and 7 species, Stemonitidaceae was the largest family $(25 \%)$ in the region.

The myxomycete samples are generally determined on dead and decaying coniferous wood, bark, leaves and debris materials (Ing and Haynes, 1999; Ergül et al., 2005; Ko et al., 2010; Baba and Özyiğit, 2017). The majority of the samples were also collected from similar substrates and in our study. In research area the percentage of lignicolous myxomycetes is $34 \%$, corticolous myxomycetes is $40 \%$, foliicolous myxomycetes is $23 \%$.

The mean number of species per genus $(\mathrm{S} / \mathrm{G})$ is 1.75 in our area. As Simberloff (1970) and others have pointed out, a low value for $\mathrm{S} / \mathrm{G}$ implies a higher overall diversity. According to Stephenson et al. (1993) this rate in Southern India is 2.24 and 4.13in North America. Alexopoulos (1970) reported that species diversity of myxomycetes is lower in tropical forests than temperate forests (Tee et al., 2014).

With this study, 28 myxomycete species were determined from Zorkun High Plateau, and a considerable contribution was made to the myxobiota of Osmaniye province and Turkey.

\section{References}

Alexopoulos CJ, Mims CW, Blackwell M (1996). Introductory Mycology. John Wiley and Sons, Inc, California Üniversity USA. Anonymous (2017). http://www.dmi.gov.tr/veridegerlendirme/il-ve-ilceler-istatistik.aspx?m=Osmaniye 
Baba H, Zumre M, Gelen, M (2016). An Investigation on North Adana (Turkey) Myxomycetes. Chiang Mai Journal of Science 43(1): 54-67.

Baba H, Özyiğit İI (2017). Three new rare Myxomycetes (Mycetozoa) records from Hatay, Turkey. Fresenius Environmental Bulletin 26(8): 4907-4910.

Ergül CC, Dülger B, Akgül H (2005). Myxomycetes of Mezit stream Valley of Turkey. Mycotaxon, 92: 239-242.

Ergül CC, Akgül H (2011). Myxomycete diversity of Uludağ national park, Turkey. Mycotaxon 116: 479.

Everhart SE, Keller HW (2008). Life history strategies of corticolous myxomycetes: the life cycle, plasmodial types, fruiting bodies, and taxonomic orders. Fungal Diversity 29: 1-16.

Gürçınar Y, Yüceer NS (1995). Çukurova Bölgesindeki yaylaların Genel Çevre Sorunları Zorkun yaylası Orneği. Çukurova Üniv, Müh. ve Mim. Dergisi 10(1,2): 79-90.

Ing B, Haynes C (1999). Corticolous myxomycetes from Belize. Kew Bulletin 54(3): 723-730.

Ko KTW, Tran HTM, Stephenson SL, Mitchell DW, Rojas C, Hyde KD, Lumyong S, Bahkali AH (2010). Myxomycetes of Thailand. Sydowia 62: 243-260.

Lado C (2005-2017). CSIC (An online nomenclatural information system of Eumycetozoa) October 20, 2017 http://www.nomen.eumycetozoa.com.

Martin GW, Alexopoulos CJ (1969). The Myxomycetes. University of Iowa, Iowa City.

Neubert H, Nowotny W, Baumann K (1993). Die Myxomyceten Vol. 1. Ceratiomyxales, Echinosteliales, Liceales und Trichiales. In verlag karlheinz baumann, gomaringen, Germany.

Neubert H, Nowotny W, Baumann K (1995). Die Myxomyceten Vol. 2. Physarales. In Verlag karlheinz baumann, gomaringen, Germany.

Neubert H, Nowotny W, Baumann K (2000). Die Myxomyceten Vol. 3 Stemonitales. In verlag karlheinz baumann, gomaringen Germany.

Sesli E, Akata I, Denchev TT, Denchev CM (2016). Myxomycetes in Turkey, a checklist. Mycobiota 6: 1-20.

Sevgi C (1984). Adana İlinin Kuzeydoğu Kesiminde yaylacılık. Ege Cografya Dergisi 2(1): 177-197.

Stephenson SL, Kalyanasundaram I, Lakhanpal TN (1993). A comparative biogeographical study of Myxomycetes in the MidAppalachians of Eastern North America and two regions of India. Journal of Biogeography 20(6): 645-657.

Stephenson SL (2014). A comparative species listing of myxomycetes from tropical (Philippines) and temperate (United States) forests. Mycosphere 5(2): 299-311.

Tee DC, Mad R, Tran HTM, Ko KTW, Stephenson SL (2014). A comparative species listing of myxomycetes from tropical (Philippines) and temperate (United States) forests. Mycosphere 5(2): 299-311.

Cite this article: Baba H (2017). Some Mycetozoa (Myxomycetes) Members from Zorkun High Plateau (Osmaniye). Anatolian Journal of Botany 1(2): 37-40. 